\title{
Die Herkunft Jahwes.
}

Von Professor Dr. Daniel Völter in Amsterdam.

Nach der israelitischen Überlieferung kann es keinem $Z$ weifel unterliegen, daß der Ursprung der Jahwereligion in der Wüste östlich von Ägypten, speziell von Gosen, und südlich von Kanaan $z u$ suchen ist. An dieses Gebiet ist Jahwe ursprünglich fest gebunden, wie sich besonders auch daraus ergibt, da $B$ noch nach der Wüstenwanderung Israels Jahwe von Seir aus den Israeliten in Kanaan zu Hilfe kommt (Jdc $5_{4}$ ), und daB selbst noch Elia von Berseba aus in vierzig Tagen und vierzig Nächten zum Gottesberg Horeb oder Sinai zieht, um eine Offenbarung Jahwes zu erlangen (I Reg $19_{38}$ ). An dieser Tatsache kann auch der Umstand nichts ändern, daß das Streben, die Mosegeschichte mit der Patriarchen- und Urvätergéschichte in Zusammenhang $z \mathrm{u}$ bringen, bei J dazu geführt hat, Jahwe als dẹn Gott der Väter Israels vorzustellen $\left(\right.$ Ex $3_{16}$ ) und seine Verehrung bis in die Urzeit zurückzuführen (Gen $4_{26}$ ). Und ebensowenig liegt ein Gegenbeweis darin, daB in einigen Nordsyrien angehörigen Namen wie Azrijau von Ja'udi und Jaubi'di der Jahwename als Bestandteil vorkommt. Denn diese Erscheinung erklärt sich am einfachsten entweder aus gelegentlicher Übertragung der Jahweyerehrung oder aber daraus, dab ein ursprünglicher mit Israel verwandter, aber versprengter Wüstenstamm in jener Gegend seßhaft geworden ist.

Im Lande Midian am Gottesberg in der Wüste soll dem dahin aus Ägypten geflohenen Mose Jahwe erschienen sein. Und zwar soll er sich ihm da geoffenbart haben im feurigen Dornbusch (Ex $3{ }_{2} \mathrm{ff}$.), weshalb Jahwe auch Dtn $33_{\text {If }}$ als der Gott, der im Dornbusch wohnt, bezeichnet wird. Der Dorn oder der Dornstrauch scheint also ein für Jahwe besonders charakteristisches Merkmal zu sein. Und es ist denn auch bezeichnend, daB innerhalb jenes Wüstengebiets im Osten Ägyptens, in das die israelitische Überlieferung den Ursprung der Jahwereligion verlegt, nicht bloß die Namen der-Wüste Zin (um Kades) und der Wüste Sin (von Elim bis zum Sinai), sondern auch des Gottesberges Sinai ge- 
funden werden, die alle mit menhangen. Es wäre unter diesen Umständen nicht zu verwundern, wenn auch das Jahwezeichen, als Zeichen des im Dornstrauch wohnenden Gottes, in einem spitzen Dreieck oder einem Dorn bestanden hätte.

Zu diesem in der Wüste östlich von Ägypten heimischen Gott Jahwe bietet die ägyptische Überlieferung eine interessante Parallele. In der ägyptischen Landschaft Gosen, die sich vom Delta ostwärts in die Wüste erstreckte, verehrte man einen Gott Har-Sopd, in welchem der lokale Gott Sopd mit dem alten ägyptischen Sonnengott Horus verschmolzen ist ${ }^{\mathrm{I}}$. Sopd, def schöne Gott vom Lande Punt, wie er in einem Texte genannt wird ${ }^{2}$, ist der Patron der Wüste im Osten Ägyptens. In der Hauptstadt der Provinz Arabia, dem heutigen Saft-el-Henneh, dem alten Per-Sopd, der Stadt des Gottes Sopd (Kesem, gr. Phakusa) im Delta am westlichen Eingang des Wadi-Tumilat hatte er ein Heiligtum ${ }^{3}$. Aber das Gebiet des Kültus dieses Gottes muß sich weit in den Osten erstreckt haben, da selbst die Inschriften in den Kupferminen und an den Felswänden der Berge auf der Sinaihalbinsel von den ältesten Zeiten an des Sopd, des Herrn des Ostens, d. h. von Arabia, gedenken*.

Das Wort spd, das dem Namen des Gottes zugrunde liegt, bedeutet eigentlich spitz sein, scharf sein, und das Zeichen des spitzen Dreiecks, das dem Gott eigen ist und mit dem sein Name. spdw geschrieben wird, stellt nach SPIEGELBERG ${ }^{5}$ ursprünglich wohl einen Dorn dar, obwohl das Wort in dieser Bedeutung bis jetzt nicht nachgewiesen ist. Die Stadt Saft, in welcher der Gott seine Kultusstätẗ hatte; wird denn auch als Hat-nebes, d. h. die Stadt des Nebes-Baums, bezeichnet, und auf den Abbildungen des Naos von Saft -aus der Zeit des Königs Nektanebos I erscheint auch der Goţt selbst in Sperbergestalt sitzend unter dem NebesBaum $^{6}$. Dieser Baum aber ist identisch mit dem Zizyphus-Lotus W., d. h. dem Lotus-Judendornbaum, der in Nordafrika wild wächst und sich vom gemeinen Judendorn durch längere Stacheln und größere Früchte (eine Art Hagebutten) unterscheidet ${ }^{7}$. Dieser unter dem LotusJudendornbaum sitzende Sopd entspricht unmittelbar dem unter dem Dornstrauch wohnenden Jahwe, und wenn der Dornstrauch, in dem Jahwe

I Ervan, Die ägypt. Rel. S. 21 ; Budge, The Gods of the Eg. I $490 \mathrm{f}$; Brugsch, Rel. u. Myth. S. $566 \mathrm{ff}$.

2 BRUGSCH, S. 676 .

2 BRugsch, S. 566 ff.; Roeder bei Roscher IV, Sp. 569.

4 BRUGSCH, S. 567; BUdGe II 290.

3 Recueil de travaux relatifs à la phil. et à l'arch. ég. XXVIII $165 \mathrm{ff}$.

- Brugsch, S. 567.569; Budge I 499.

? Baedeker, Ägypten, 4. A. S. LXXVI. 
dem Mose erscheint, feurig ist, so erklärt sich dies gerade von Sopd aus, da dieser die Sonnenscheibe auf dem Haupte trägt ${ }^{\mathrm{x}}$, wie ja auch der ihm verwandte oder in sein Erbe eingetretene Rê Harachte auf der Abbildung bei BUDGE ${ }^{2}$ mit der Sonnenscheibe auf dem Haupt unter dem Nebes-Baum dargestellt ist. Wenn aber Jahwe gerade in der Wüste am Gottesberg im Osten dem Mose unter dem feurigen Dornbusch erscheint oder von Mose da gleichsam gefunden wird, so ist von dem mit Sopd identifizierten Horus wesentlich dasselbe zu sagen, da die Wüste am Eingang von Punt oder das Ostgebirge der Fundort des Horusauges ist ${ }^{3}$, das hier dem Sonnenauge entspricht ${ }^{4}$. .

Zwischen Jahwe und Sopd, beziehungsweise Har-Sopd ergibt sich also auf Grund des Gesagten die merkwürdigste Übereinstimmung. Und da auf genau demselben Gebiet zu gleicher Zeit nicht zwei Götter von ganz derselben Art nebeneinander bestanden haben können, so kommt man notwendig zu dem Schluß, daß Jahwe und Sopd miteinander identisch sein müssen. Die Identität derselben läßt sich denn auch durch eine nähere Vergleichung beider noch weiter erhärten.

Nach seinem ursprünglichen Wesen ist Jahwe allem nach ein Sonnengott gewesen. Das beweisen nicht bloß die Worte in Dtn $33_{2}$ : ¿Jahwe kam vom Sinai her und glänzte ihnen auf von Seir; er ließ sein Licht leuchten vom Gebirge Paran", sondern das ergibt sich auch schon daraus, da $B$ Jahwe im feurigen Dornbusch, d. h. als die im Dornbusch sich befindende Sonnenscheibe, sich dem Mose offenbart. Geradeso ist auch der unter dem Nebes-Baum sitzende Sopd ein Sonnengott, der als solcher die Sonnenscheibe auf dem Haupte trägt. Und auch das Zeichen des Dreiecks oder Dorns, das Sopd bezeichnet, zeugt für den Sonnencharakter desselben, da dieses Zeichen, das auch dem Sothisstern, d. h. dem strahlenden Sirius, eigen ist, wohl nichts anderes ist als ein Symbol der Strahlen, die von der Sonnenscheibe ausgehen. Bei Sopd sowohl als bei Jahwe deutet also der Dornbusch oder Dornbaum mit seinen Stacheln ohne Zweifel das Strahlenbüschel der Sonne an.

Wenn es in Jdc $5_{31}$ heiBt, daß diejenigen, die Jahwe lieben, seien wie der Aufgang der Sonne in ihrer Pracht, so könnte man daraus schließen, daß Jahwe besonders die aufgehende Sonne repräsentiert. Von Sopd gilt dies ebenfalls. Auch er ist wohl speziell der Gott der aufgehenden Sonne. Das ergibt sich nicht bloß daraus, daß er bezeichnet

1 BRUGSCH, S. 566 .

2 Budge, I 474, vgl. dazu S. 479.

3. Sethe, Zur altägypt. Sage vom Sonnenauge, das in der Fremde war. Leipzig, Hinrichs 1912, S. 14. 16. 21. 22. 24. 25. 33. 39. 40.

4 Ebenda, S. 33. 37. 38. 
wird als der schöne Gott vom Lande Punt, d. h. von dem fernen Ostland zu beiden Seiten des Roten Meeres, sondern auch und besonders daraus, da $B$ er mit Horus verschmolzen und darum auch der Horus des Ostens genannt wirdx. Denn auch Horus „der Strahlensender, der aus dem Horizont hervorgeht " 2 , ist der Gott der aufsteigenden, jugendlichen Sonne ${ }^{3}$. Und da der Himmel bei Tag das "Angesicht" des Horus ist", und dieser selbst wie auch Sopd ${ }^{5}$ der Gott mit dem leuchtenden Augenpaar ist, so ergibt sich von da aus noch eine weitere Parallele, sofern auch bei den Israeliten nicht bloß von Jahwes "Angesicht" geredet wird, das Israel nach seinem Bestimmungsort begleiten soll (Ex 33 I2-I5; vgl. Jes 63 ,), sondern auch von Jahwes Augen, die beständig auf das Land Israel gerichtet sind, vom Anfang bis zum Ende des Jahres (Dtn II ${ }_{11}{ }_{12}$ ).

Für Jahwe ist nun aber weiter besonders charakteristisch seine enge Beziehung zum Gottesberg Sinai oder Horeb, sei's, daß er am Himmel über dem Berge wohnt und der letztere nur die vornehmste Stätte seiner Offenbarung ist, sei's, daB der Berg selbst als sein Wohnplatz gedacht ist (Ex 3 I8 19202433 34).

Die alttestamentliche Überlieferung läßt uns freilich hinsichtlich des Gottesberges sehr im Unsichern. GewiB.ist eigentlich nur, daß es ein Berg im Osten von Ägypten ist. Was seinen Namen betrifft, so nennt ihn der Elohist und der Deuteronomiker Horeb, aber der die älteste Überlieferung vertretende Jahwist, wie auch der Priesterkodex, nennt ihn Sinai. Dieser Name weist an sich schon am wahrscheinlichsten auf den-gewaltigen Gebirgsstock auf der Halbinsel zwischen den beiden Busen des Roten Meeres, mit dem nun einmal der Name Sinai verbunden ist. Und daB die Israeliten am Gottesberg in der Wüste mit den Kenitern in Vérbindung traten (Num io ${ }_{29} \mathrm{ff}$. Jdc $\mathrm{I}_{16} 4_{\mathrm{II}}$ ), ist auch ein Beweis dafür, daB man beim Gottesberg an den Sinai in der gewöhnlichen Bedeutung dieses Namens zu denken hat. Denn die Keniter waren, wie ihr Name andeutet, ein midianitischer Stamm, der das Schmiedehandwerk betrieb, und für Schmiede oder Erzarbeiter war der Sinai seiner Kupferminen wegen natürlich von alters her ein gesuchter Ort.

Freilich scheint manches in unserer Überlieferung dafür zu sprechen, daB der Gottesberg Sinai oder Horeb ein Berg westlich oder östlich von Kades gewesen ist. Aber die unklaren und widerspruchsvollen Detail-

1 Brugsch, Rel. u. Myth., S. 568. 630; Ägyptologie, S. 452; Wiedemans, D. Rel. d. alten Ägypt., S. 87.

2 WIEDEMANN, S. 42.

I Ed. Meyer, Art. Horus bei Roscher, I 2, Sp. 2744 ff.

4 Budge, I 466 ff.; Lange bei Saussaye 2, I, S. 145.

5 BRUGSCH, S. 568.

Zeitschr. f. d. alttest. Wiss. Jahrg. 37. 1917/88. 
angaben über den Wüstenzug der Israeliten haben wenig Wert. Es liegt ihnen die ohne $Z$ weifel falsche Vorstellung zugrunde, daE die Israeliten aus Ägypten ausgezogen seien, um zum Gottesberg in der Wüste und dann weiter nach Kanaan zu ziehen. Das führte von selbst dazu, den Gottesberg Ägypten näher zu rücken oder jedenfalls in der Richtung nach dem hl. Lande zu suchen. Die Wahrheit wird die sein, dab die israelitischen Stämme Nomaden waren, die in der Wüste zwischen Gosen und dem Sinai umherzogen, wohl auch bis zum Sinai selbst oder wenigstens in seine Nähe kamen und schlieBlich von Kades aus nach Palästina vordrangen.

$\mathrm{Da} B$ in der Tat der Gottesberg der Israeliten der auf der Halbinsel gleichen Namens gelegene Sinai gewesen sein muB, ergibt sich mit besonderer Evidenz gerade aus der Identität von Jahwe und Sopd. Denn auch Sopd war ein am Sinai verehrter Gott, wie die Inschriften in den Kupferminen und an den Felswänden daselbst von den ältesten Zeiten an bezeugen. Die enge Beziehung des Sopd als des Gottes der aufgehenden Sonne zum Sinai ist denn auch sehr begreiflich, da die Schutzgottheit oder die Herrin der Māfekt-Gegend, d. h. der Sinai-Gegend, die Hathor ist ${ }^{I}$, die Mutter der Sonne, die Mutter speziell des Horus und des Sopd ${ }^{2}$, die Göttin, die wie im Westen zum Berg des Sonnenuntergangs $^{3}$, so im Osten zum Berg des Sonnenaufgangs in Beziehung gestanden zu haben scheint ${ }^{4}$. Dazu stimmt durchaus, daß auch nach der israelitischen Auffassung, wie sie noch in jenen bereits zitierten Worten aus Dtn $33_{2}$ zutage tritt, der Sinai der Berg des Sonnenaufgangs ist.

Wir haben nun aber die Verwandtschaft des Jahwe und Sopd noch weiter zu verfolgen. Jahwe ist nach der israelitischen Überlieferung der Gott, der des Nachts in der Feuersäule, bei Tag in der Wolkensäule vor Israel herzieht (Ex $3_{2 \mathrm{I}}$ ): Was zunächst die Feuersäule betrifft, so liegt nichts näher, als hiebei' an 'das Zodiakallicht zu denken, das wie eine patronenförmig am Horizont aufsteigende Säule in den Tropen die Nacht mit seinem milden Glanz erleuchtet. Wenn nach der Erzählung vom Durchzug durchs Rote Meer statt der.Wolkensäule, die den Israeliten bei Tàg voranzog, die Feuersäule bei Anbruch der Nacht hinter den

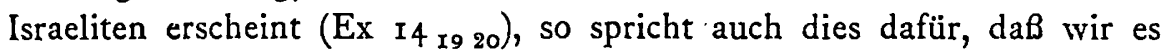
dabei mit dem Zodiakallicht zu tun haben, da dasselbe nach der Abenddämmerung am westlichen Himmel zu sehen ist.

I Vgl. über die Zeugnisse vom Sinai L. Eckenstern, Moon-Cult in Sinai usw. Ancient Egypt. I 9 ff. Überdies Turajerf, Die naophore Statue Nr. 97 im Vatikan, ZÄSA 46, S. $74 \mathrm{ff}$.

2 Brugsch, S. 569 . 3 ERMan, S. 12.

4. VIREY, La religion de l'ancienne Égypte, Paris 1910, S. 185. 
Repräsentiert nun Jahwe das Zodiakallicht, so tut dies Sopd ohne Zweifel auch. Manche Ägyptologen haben selbst gemeint, daß Sopd nichts anderes als der Gott des Zodiakallichts gewesen sei. Doch ist das nicht richtig. Aber das Zodiakallicht gehört dem Sopd jedenfalls zu. Denn auf dem Schrein aus seinem Kultusort Saft el-Henneh ist, wie auch SPIEGELBERG anerkennt, zweifellos das Zodiakallicht abgebildet. Patronenförmig erhebt es sich da am Horizont unter dem gestirnten Himmel, während sich der Pharao davor anbetend zur Erde niedergeworfen hat ${ }^{\mathrm{I}}$. $\mathrm{Da}$ das Zodiakallicht wie ein Lichtberg, so glänzend wie die Milchstraße des Himmels oder wohl noch glänzender als diese, am Horizonte aufsteigt, so ließe sich hiemit vielleicht auch die Erzählung in Ex $24_{9-1 \text { ir }}$ in Zusammenhang bringen, wonach Mose und siebzig Älteste auf den Berg stiegen, wo sie den Gott Jahwe sahen, zu dessen Füßen es sich wie aus Saphirplatten aufbaute, so glänzend hell wie der Himmel selbst. Man müBte dann nur Mose auf den Mond und die Ältesten auf die Sterne deuten, was bei Mose, wie ich nachzuweisen gesucht habe, durchaus angezeigt ist ${ }^{2}$. Doch kann man bei der Erzählung auch an einen Besuch auf dem Sinai-Ostberg denken und ein Text auf dem Naos zu el-Arisch liefert dazu sogar eine gewisse Parallele, sofern nach diesem Texte Schu mit seinem Gefolge nach dem Berg am Osthorizont zieht, um seinen Vater Rê Harachte zu sehen ${ }^{3}$.

Wie zur Feuersäule Jahwes bei Nacht, so bietet nun Sopd auch eine Parallele zu der Wolkensäule Jahwes bei Tag. Ja auf diese Erscheinung fällt erst von Sopd aus das rechte Licht. Sopd wird nämlich wie mit Horus so auch mit Schu identifiziert ${ }^{4}$. Er wird darum nicht bloß als Har-Sopd, sondern auch als Sopd-Schu bezeichnet. Dieser Schu ist auch ein alter Sonnengott, ' und zwar ist er speziell der von den Sonnenstrahlen durchleuchtete Luft- und Wolkenraum, der sich bei Tag wie eine Säule $z$ wischen Erde und Himmel stellt ${ }^{5}$. Hier haben wir offenbar die Parallele, und zwar die erklärende Parallele zu der Wolkensäule, in der Jahwe bei Tag vor Israel herzieht. Aber auch daß Jahwe gelegentlich in die Wolke sich hüllt oder in der Wolke erscheint, erklärt sich aus der Beziehung des Schu, beziehungsweise Sopd-Schu zu dem von ihm durchleuchteten Luft- und Wolkenraum, "zumal da die "Stützen"

- Vgl. die Abbildung bei Brugsch, PSBA XV 39i und dazu Spregelberg, aaO. I66.

2 VgI. dazu meine Schrift: Jahwe und Mose, Leiden, Brill igr4.

3 Vgl. Roeder, Art. Schow bei Roscher; IV, Sp. 569. Der Naos von el-Arisch stammte vielleicht aus Saft el-Henneh.

4 Brugsch, Mytb.; S. 566. 569-571; Ägyptologie, S. 452; Roeder bei Roscher, aaO., Sp. $566.575 \cdot 576.578$.

s ErMan, S. 15; Brugsch, S. 431 usw.; Roeder bei Roscher IV, Sp. 57 I. 
des Schu, jedenfalls in der späteren Zeit, auf die Wolken scheinen gedeutet $z \mathrm{u}$ werden ${ }^{\mathrm{x}}$. Und da Schu auch der Gott ist, der in Sturm und Unwetter sich offenbart und Himmel und Meer in Aufruhr zu versetzen vermag ${ }^{2}$, so entspricht er auch damit dem im Gewitter unter Donner und Blitz auf den Sinai herunterfahrenden oder in träufenden Wetterwolken von Seir kommenden Jahwe. Endlich aber stimmen Jahwe und Sopd auch darin liberein, daB beide Kriegsgötter sind, wie es sich von der die Mächte der Finsternis vertreibenden aufgehenden Sonne von selbst versteht. Wie Jahwe speziell nach $\mathrm{Ex}_{\mathrm{I}_{3}}$ und Jdc $5_{4}$ s ein Kriegsmann und Kämpfer ist, so ist es auch Sopd (Har-Sopd), denn dieser ist der "Herr der Schlacht", der "Schläger der Bergvölker" 3 .

Die Frage, die uns noch übrig bleibt, ist nun aber die, wie es kommt, daß die beiden einander só völlig entsprechenden Götter einen verschiedenen Namen tragen. Bei den Ägyptern heißt der Gott Sopd, bei den Israeliten Jahwe. Aber wie der ägyptische Name Sopd den Gott nach einer auch den Israeliten bekannten Eigenschaft desselben, d. h. nach seinem Symbol, dem Dorn, bezeichnet, so bezeichnet der israelitische Name Jahwe den Gott nach seinem auch den Ägyptern bekannten Wesen. Denn der ägyptische Sopd entspricht als Gott der aufgehenden Sonne dem ägyptischen Chepera und kann Chepera genannt werden, sofern dieser Name eben die ägyptische Bezeichnung für die aufsteigende Sonne oder die Morgensonne ist. Chepera aber bedeutet "der Werdende"4. Leitet man nun den Namen Jahwe, wie in Ex $3_{\text {I }}$ geschieht, ab von $=$ = werden, so scheint der israelitische Name Jahwe dasselbe auszudrücken wie der ägyptische Name Chepera und ebenfalls eine Bezeichnung für die Morgensonne zu sein.

Wir meinen auf Grund der vorhergehenden Darlegungen Recht genug $z u$ haben, um anzunehmen, daß der ägyptische Sopd und der israelitische Jahwe trotz der Verschiedenheit der Bezeichnung ein und derselbe Gott sind. Sie decken sich ja vollkommen sowohl nach ihren Eigenschaften als nach dem Gebiet, auf dem sie zu Hause-sind. Damit soll aber nicht behauptet werden, daß die Israeliten einfach einen ägyptischen Gott übernommen haben. Sopd ist vielmehr sehr wahrscheinlich ursprünglich gar kein ägyptischer Gott gewesen. Er ist wohl von den Ägyptern übernommen worden, aber im eigentlichen Ägypten, abgesehen vom östlichen Delta, nicht eigentlich heimisch geworden. BUDGE meint, daß der

I ROEDER, aaO. Sp. 572.

${ }^{2}$ MASPero, Hist. anc. I, S. i70. 178. . Roeder bei Roscher IV, Sp. 570.571.

3 Budge I 498. 499; Brugsch, S. 566. 568. 569: WiedemanN, S. 87.

4 Wiedemanin, D. Rel. d. alten Äg., S. I7; Zmmermann, D. äg. Rel., S. I29. 
Gott aus. Syrien stamme. Doch läßt sich das nicht beweisen. Wahrscheinlich ist er der Gott der semitischen Nomaden in der Wüste zwischen Gosen und dem Sinai gewesen, und zu diesen Nomaden gehörten die israelitischen Stämme. Ist Sopd, beziehungsweise Jahwe aber auch ursprünglich der Gott dieser Wüstenbewohner gewesen, so hat er doch nicht bloß bei den Ägyptern Aufnahme gefunden, sondern ist umgekehrt wieder der Einfluß Ägyptens auf die östliche Wüste groß genug gewesen, um auch dem Gott dieser Wüste mehr oder weniger den Charakter eines ägyptischen Gottes aufzudrücken. Aber das Bewußtsein, daß es ihr Gott ist, wird bei jenen Nomaden lebendig geblieben sein und damit auch das Bewußtsein ihres Unterschiedes von oder ihres Gegensatzes gegen Ägypten.

Zur Vollständigkeit unserer Beweisführung gehört nun freilich auch noch die Beantwortung der Frage, wer Mose gewesen ist, und wie es kommt, daß Mose zu diesem Gott Jahwe = Sopd in so enger Beziehung steht. Aber dafür kann ich auf meine Schrift Jahwe und Mose ${ }^{\mathrm{I}}$ verweisen.

I Brill, Leiden 1914, S. 10-45. 